\title{
Core Language of Thought
}

\author{
Kyo Takano \\ Independent Researcher \\ Japan \\ kyo.psych@gmail.com
}

\begin{abstract}
Fodor's "Language of Thought" (also called Mentalese) hypothesis - that we use a language-like, syntactic system in processing thoughts instead of natural languages - caused controversies in philosophy, psychology, and linguistics. While many researchers proposed their perspectives, both for and against, none of them addressed its evolutionary and sociocultural variabilities as far as I know. Accordingly, I provide a novel perspective on the hypothesis by discussing the interplay among our cognition, natural languages, evolution, and sociocultural factors. First, like one speaks personalized English as his/her idiolect, individuals have their own "Idiolects of Thought" as a derivative of Mentalese. Second, while Mentalese varies from individual to individual, "Core Language of Thought" is cognitively fundamental and largely shared across individuals.
\end{abstract}

While people often think they use English or other natural languages, it is not a plausible explanation of our thought processes. A language is itself is a mere medium to represent thoughts comprised of their constituent concepts, and our brains enable encoding/decoding of neural representations corresponding to such. Instead, like infants without words can make simple inferences, it is more plausible to believe we possess a mind-internal system-probably innate- to process thoughts.

Fodor (Fodor 1975; 2008) named such a system the "Language of Thought" (also Mentalese). The Language of Thought hypothesis states that thoughts are held and processed in Mentalese, a system that has a language-like syntax and compositional semantics. Like natural languages, a Mentalese sentence in Fodor's definition supposedly consists of token representations of concepts, and its meaning is systematically determined by those representations and the way they are composed together. For example, an English phrase YOUR DOG RAN AWAY can denote that a specific dog in your house ran away to somewhere, as the meaning can be reconstructed to its mentalese expressions through corresponding mental representations of YOUR, DOG, RAN AWAY, and the subject-verb structure.

Although many scholars-philosophers, psychologists, and linguists - debated over this controversial hypothesis, to my knowledge, there has been no argument concerned with
Mentalese's generational or interpersonal variabilities. Accordingly, in this article, I present a novel perspective on the Language of Thought hypothesis, incorporating such variabilities caused by several factors. Specifically, I argue that individuals have their own "Idiolects of Thought," which partly vary from individual to individual and share a large portion of common features that I would call the "Core Language of Thought." This contrast is equivalent to the fact that, for example, all English speakers do not use exactly the same English but their idiolects-English with slight and unique variations. In providing my argument, first, I will present premises for my argument from evolutionary, sociocultural, and cognitive perspectives. Subsequently, I present how humans can have variational Mentalese, a language-like system to process mental representations of concepts, both evolutionarily and socioculturally with illustrations.

\section{Premises}

\section{Connectionism}

In discussing the Language of Thought hypothesis, as a premise, I take a connectionist approach and regard that concepts in thoughts are distributed representations in the brain. Here, I interpret Mentalese as a metaphorical projection of how concepts are neurally and thus high-dimensionally represented and processed; in other words, Mentalese is fundamentally rooted in neural representations of concepts, and their relations can still assume it in the form of language. Although Fodor and some scholars disagree with the connectionist view, particularly because it seems to undermine the systematicity of Mentalese as an information-processing system (Fodor and McLaughlin 1990; Fodor, Pylyshyn, and others 1988), I refute this objection for the following reasons. First, Mentalese is just a language-like syntactic system but an actual language. While natural language expressions can be analyzed and explained, that is only because our linguistic system can decode them into Mentalese expressions, and thus Mentalese itself need not necessarily have this property of natural language. Second, such distributed representations do not undermine the systematicity of thoughts, whereas enjoying flexibility and productivity. While an explainable system would feel intuitively systematic, Mentalese neither equals natural languages nor has to 
be as highly explainable as English. Simultaneously, systematicity can be achieved without high explainability when nodes of mental representations can constitute a thought state in a structure-sensitive manner (Chalmers 1990; 1992; Niklasson and Van Gelder 1994; Markman and Dietrich 2000). Finally, connectionism also explains various mental representations/processes, including concepts that words can denote and logical structure, perception, mental map, etc. (Rescorla 2019). For more details of the controversy, see the review by Rescorla (2019).

\section{Brain-Language Interaction}

Second, our cognition and natural languages are strongly dependent on each other; the usage of natural languages influence the way we think (language relativity) (Kay and Kempton 1984; Hussein 2012; Boroditsky 2001; Alderson-Day and Fernyhough 2015), and the way we think shapes our use of natural languages. One explanation for such a mutual dependence is that, over generations, languages evolved to convey more and more detailed social messages as a brain-friendly communication tool, which required humans to have language-friendly cognitive functions (Evans and Levinson 2009).

\section{Translanguaging}

Finally, I also assume that humans have innate cognitive capacities to learn human-level languages and that learning/acquiring a language involves lots of factors including but not limited to linguistic, cognitive, and semiotic ones, as Wei (2018) argues. This is the concept of "Translanguaging" (Wei 2018), which allows language users to constitute their own idiolects by integrating different dimensions associated with their social lives, including native language, ideology, personal experiences, etc., beyond the linguistic domain. Put another way, for example, one's native language is a mere determinant of his/her idiolect, among many other factors. Since the concept well explains the sociocultural variability of natural language and mentalese, I will employ the Translanguaging perspective in expanding my argument. Here, although Wei (2018) opposed Fodor's Language of Thought argument (1975), I do not fully accept the rejection because it was largely based on the assumption that Mentalese exists independently of idiolects; in my argument, I allow Mentalese to have variability associated with idiolects. Translanguaging is also highly compatible with the above two premises. First, the connectionist view assumes distributed representations of thoughts and thus language use; this suggests that the use and acquisition of languages can plausibly extend to other interrelated cognitive domains. Second, Translanguaging explains intra-generational interaction between brain and language when there is little literature on the evolutionary interaction.

\section{Core Language of Thought}

Having provided these premises, I propose that we have variational languages of thought-which I call "Idiolects of Thought" — and that a significant proportion of it is cross-personally consistent as "Core Language of Thought,"
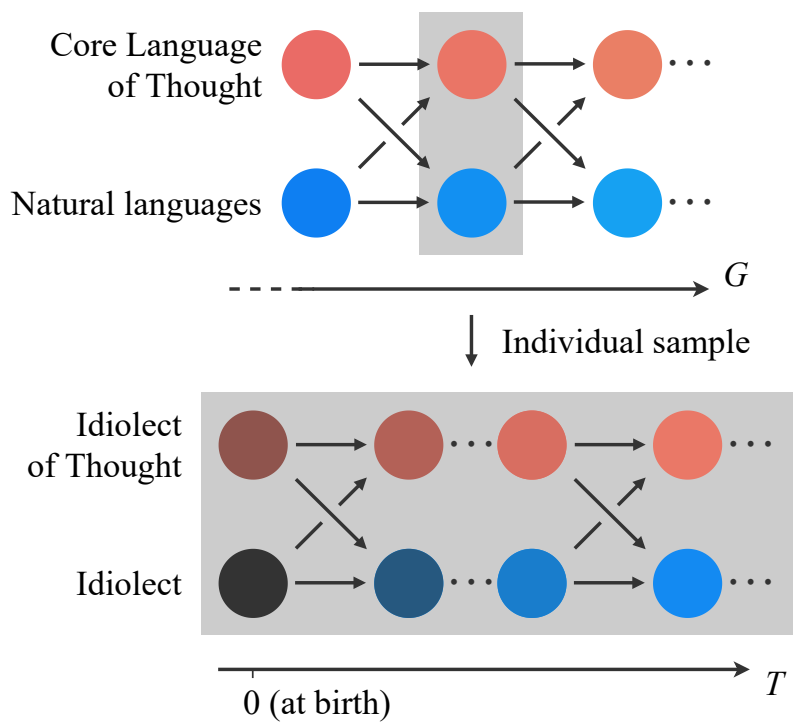

Figure 1: Evolutionary and Translanguaging variations of Language of Thought, with a constant environment assumed. The upper half depicts the slight differentiations of both the Core Language of Thought and natural languages influencing each other over generations. The bottom half shows how an individual shapes his/her Idiolect of Thought and natural language(s) together. With innate neural registers for certain primary concepts, s/he learns to use natural languages as his/her idiolect, which mutually influences his/her idiolect of thought.

which I consider is the basis of Mentalese and also is largely innate. Like every English speaker uses personalized English slightly deviated from other English speakers, I suggest that Mentalese be considered in the same way; in other words, Mentalese does not have to be totally consistent regardless of thinkers as mentioned above. At the same time, while our Mentalese varies from individual to individual, a large part of it is plausibly shared across individuals. This is because we share fundamentally the same cognitive features shaped by evolutions. Borrowing Wei (2018)'s perspective of Translanguaging, the variability of Mentalese can be considered largely caused by a number of sociocultural factors, such as languages used in one's everyday life, ideology, personal experiences, cognitive capacities, etc., like Wei (2018) addressed how individuals learn to have idiolects as a result of Translanguaging. Figure 1 metaphorically illustrates evolutionary transformation and intra-generational variability of Mentalese. Core Language of Thought, as the representative component of Mentalese, and natural languages are fairly stable over generations; however, they still have slight effects on each other in response to natural languages' social utility, brain-language fit, etc. In contrast, one individual's Idiolect and Idiolect of Thought grows rapidly; at birth, even with no utterance ability, s/he expectedly has his/her own immature Idiolect of Thought as a derivative of Core Language of Thought, which is associated with the innate capacity to learn natural languages. Over the lifetime, the 


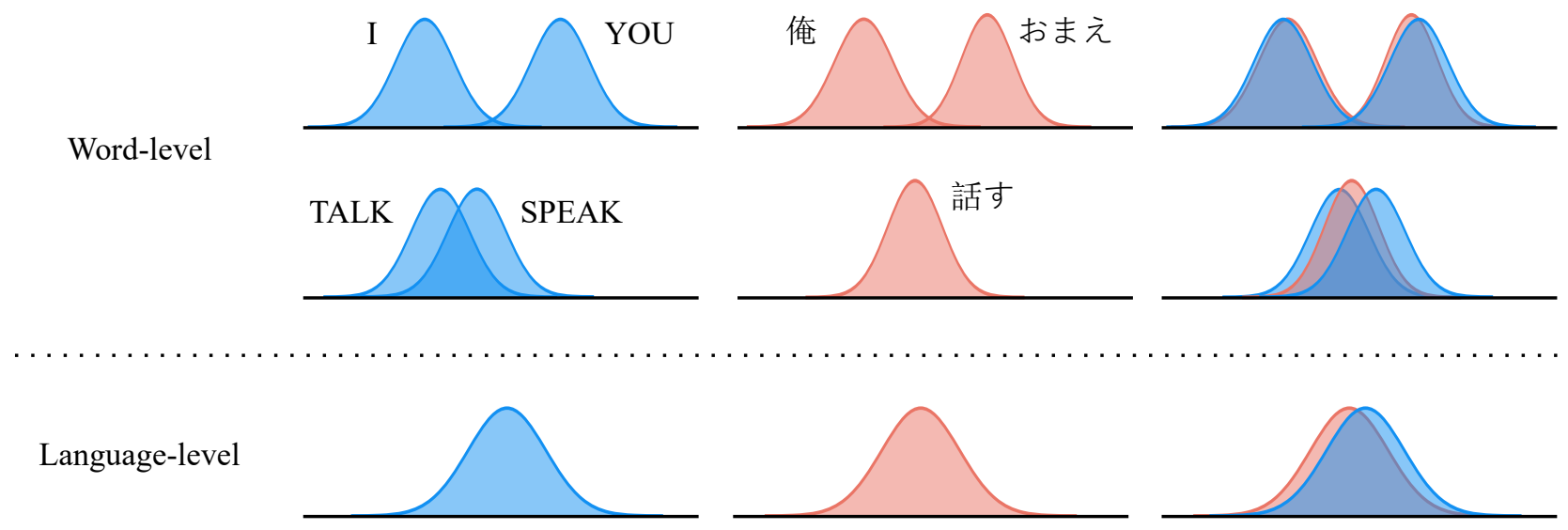

Figure 2: Pseudo feature embeddings of different Idiolects of Thought into 1D space on both word and language levels. Each distribution curve represents a concept or a natural language with contextual variances in one's Idiolect of Thought, and they share common and context-dependent features over the overlapping areas. For the word-level embeddings, the top row shows fairly distinct concepts of single- and second-person nouns, which are probably innate; they overlap only slightly within each individual's idiolect of thought, while there are also little differences between individuals. On the other hand, the second row indicates that even if there are some deviations between concepts, they also share certain features. As a paradigm of such perspectives, it could be inferred that languages also share certain features independent of individuals who speak different idiolects and have different Idiolects of Thought.

individual learns to speak natural languages in the form of idiolect while also developing Idiolect of Thought in the mutual relationship.

To further address how Idiolects of Thought vary across individuals, I metaphorically depict translanguaging variability of neural representations in Figure 2. Although this illustration lacks authenticity as words and languages cannot be embedded into such a low dimension realistically, it visualizes qualitative similarities/differences of their neural representations both intra- and inter-personally. In the top example, concepts like I and YOU are very distinct as they are plausibly determined by our evolutionary need to distinguish the most important communication entities. This example is certainly cross-culturally consistent like the Japanese language has its equivalents. Accordingly, when overlaid, there are only small interpersonal gaps for neural representations of these concepts. On the other hand, TALK, SPEAK, and their Japanese equivalent have similar but slightly different connotations. Regarding these gaps, I would explain that the overlapping distribution of features represents constituent concepts shared by these words' neural representations and that the differences are a product of slight differences of translanguaging experiences. Finally, summing up these observations, I contend that different individuals - either from the same or different cultures-fundamentally consist of largely the same features. Even though some neural representations for equivalent concepts might seem somewhat different like in the second row, such concepts and their corresponding neural representations are consistent across individuals from different translanguaging experiences when reduced to their lower-level constituents.

As a limitation, this argument can be true if and only if the variabilities of Mentalese are accepted, and also our cognitive characteristics are, in fact, fairly the same from individual to individual. Although I have no means to prove these premises, based on my introspection, they are intuitive and make the Language of Thought hypothesis more plausible as a theory of our thought processes. Because the purpose of this paper is to present the Core Language of Thought and Idiolects of Thought, I did not fully address past controversies on Metalaese.

\section{Conclusion}

Discussing the nature of natural languages and Mentalese from evolutionary, sociocultural, and cognitive perspectives, in this paper, I proposed that every individual have his/her own Idiolect of Thought as a derivative of Mentalese while sharing the Core Language of Thought with others as a fundamental component of Mentalese. As far as I know, this paper is the first to assess Mentalese-also called the Language of Thought hypothesis - on its evolutionary and sociocultural variabilities together with those of natural languages. The variability of Mentalese might have implications for several fields such as linguistics, cognitive psychology, Natural Language Processing (a branch of artificial intelligence), language education, etc. 


\section{References}

Alderson-Day, B., and Fernyhough, C. 2015. Inner speech: development, cognitive functions, phenomenology, and neurobiology. Psychological Bulletin 141(5):931.

Boroditsky, L. 2001. Does language shape thought?: Mandarin and english speakers' conceptions of time. Cognitive Psychology 43(1):1-22.

Chalmers, D. 1990. Why fodor and pylyshyn were wrong: The simplest refutation. In Proceedings of the Twelfth Annual Conference of the Cognitive Science Society, Cambridge, Mass, 340-347.

Chalmers, D. J. 1992. Syntactic transformations on distributed representations. In Connectionist Natural Language Processing. Springer. 46-55.

Evans, N., and Levinson, S. C. 2009. The myth of language universals: Language diversity and its importance for cognitive science. Behavioral and Brain Sciences 32(5):429-448.

Fodor, J., and McLaughlin, B. P. 1990. Connectionism and the problem of systematicity: Why smolensky's solution doesn't work. Cognition 35(2):183-204.

Fodor, J. A.; Pylyshyn, Z. W.; et al. 1988. Connectionism and cognitive architecture: A critical analysis. Cognition 28(1-2):3-71.

Fodor, J. A. 1975. The language of thought, volume 5. Harvard University Press.

Fodor, J. A. 2008. LOT 2: The language of thought revisited. Oxford University Press on Demand.

Hussein, B. A.-S. 2012. The sapir-whorf hypothesis today. Theory and Practice in Language Studies 2(3):642-646.

Kay, P., and Kempton, W. 1984. What is the sapir-whorf hypothesis? American Anthropologist 86(1):65-79.

Markman, A. B., and Dietrich, E. 2000. In defense of representation. Cognitive Psychology 40(2):138-171.

Niklasson, L. F., and Van Gelder, T. 1994. On being systematically connectionist. Mind and Language 9(3):288-302.

Rescorla, M. 2019. The language of thought hypothesis. https://plato.stanford.edu/entries/language-thought/. Accessed: 2021-01-12.

Wei, L. 2018. Translanguaging as a practical theory of language. Applied Linguistics 39(1):9-30. 